\title{
High Resolution Manometry Analysis of a Patient With Dysphagia After Occiput-C3/4 Posterior Fusion Operation
}

\author{
Yoongul Oh, $\mathrm{MD}^{1}$, Seok Tae Lee, $\mathrm{MD}^{1}$, Ju Seok Ryu, MD, $\mathrm{PhD}^{2}$ \\ ${ }^{1}$ Department of Rehabilitation Medicine, CHA Bundang Medical Center, CHA University, Seongnam; \\ ${ }^{2}$ Department of Rehabilitation Medicine, Seoul National University Bundang Hospital, \\ Seoul National University College of Medicine, Seongnam, Korea
}

\begin{abstract}
Many reports of changes in cervical alignment after posterior occipitocervical (O-C) fusion causing dysphagia are available. The clinical course can range from mild discomfort to severe aspiration. However, the underlying pathogenesis is not well known. We report an 80-year-old female with videofluoroscopic swallowing study evidence of aspiration that developed after occiput-C3/4 posterior fusion. Pharyngeal pressure was analyzed using high resolution manometry (HRM). Impaired upper esophageal sphincter opening along with diminished peristalsis and pharyngeal pressure gradient were revealed by HRM to be the main characteristics in such patients. The patient fully recovered after a revision operation for cervical angle correction. Distinct pressure patterns behind reversible dysphagia caused by a change in cervical alignment were confirmed using HRM analysis.
\end{abstract}

Keywords Manometry, Deglutition disorders, Cervical vertebrae, Neurosurgery

\section{INTRODUCTION}

Changes in cervical alignment are proposed to be a cause of dysphagia. Numerous cases of dysphagia after posterior occipitocervical (O-C) fusion have been reported [1-4]. Previous studies have suggested that a decrease in the extension of the $\mathrm{O}-\mathrm{C} 2$ angle is the cause of dyspha-

Received January 5, 2015; Accepted March 11, 2015

Corresponding author: Ju Seok Ryu

Department of Rehabilitation Medicine, Seoul National University Bundang Hospital, Seoul National University College of Medicine, 82 Gumiro 173beon-gil, Bundang-gu, Seongnam 13620, Korea

Tel: +82-31-787-7739, Fax: +82-31-787-4051, E-mail: jseok337@snu.ac.kr

(c) This is an open-access article distributed under the terms of the Creative Commons Attribution Non-Commercial License (http://creativecommons. org/licenses/by-nc/4.0) which permits unrestricted noncommercial use, distribution, and reproduction in any medium, provided the original work is properly cited.

Copyright (C) 2015 by Korean Academy of Rehabilitation Medicine gia in such patients [1-4]. However, the underlying pathophysiology remains unknown, and direct evidence of the disturbances in pharyngeal pressure generation has not been studied [2].

Recently, high resolution manometry (HRM) was developed and started being used for the evaluation of pharyngeal dysphagia. HRM has pressure sensors at 1- to 2-cm intervals and uses a solid catheter, which enables evaluation in the sitting position. As HRM provides information about precise pharyngeal pressure events, it can be used to study the mechanism of dysphagia with regard to pressure generation.

We report a patient who developed dysphagia after posterior occiput-C3/4 fusion (O-C3/4 PF), and distinctive abnormalities in pressure generation were confirmed by HRM. This is the first report of changes in a patient with dysphagia after cervical misalignment, measured by 
HRM.

\section{CASE REPORT}

An 80-year-old female with a 20-year history of hypertension and diabetes visited the Department of Neurosurgery at the CHA Bundang Medical Center. Her complaint was aggravation of chronic posterior neck pain in the previous 4 months. C-spine magnetic resonance imaging revealed a retro-odontoid pseudotumor between the $\mathrm{C} 1$ and 2 levels. To solve the problem, $\mathrm{O}-\mathrm{C} 3 / 4 \mathrm{PF}$ with $\mathrm{C} 1$ total laminectomy and removal of adhesive tissue for cord decompression was completed on December 10, 2013.

The patient complained of dysphagia and sore throat after the operation. Dysphagia persisted for more than 10 days. She was referred to the department of otolaryngology for laryngoscopic evaluation. Upon examination, only aphthous ulcers were noted while vocal cord movements were intact. Though she received conservative treatment, dysphagia persisted despite alleviation of the sore throat. The patient was referred to the department of rehabilitation on January 2, 2014.

The O-C2 angle at the neutral position was $5.2^{\circ}$ in the
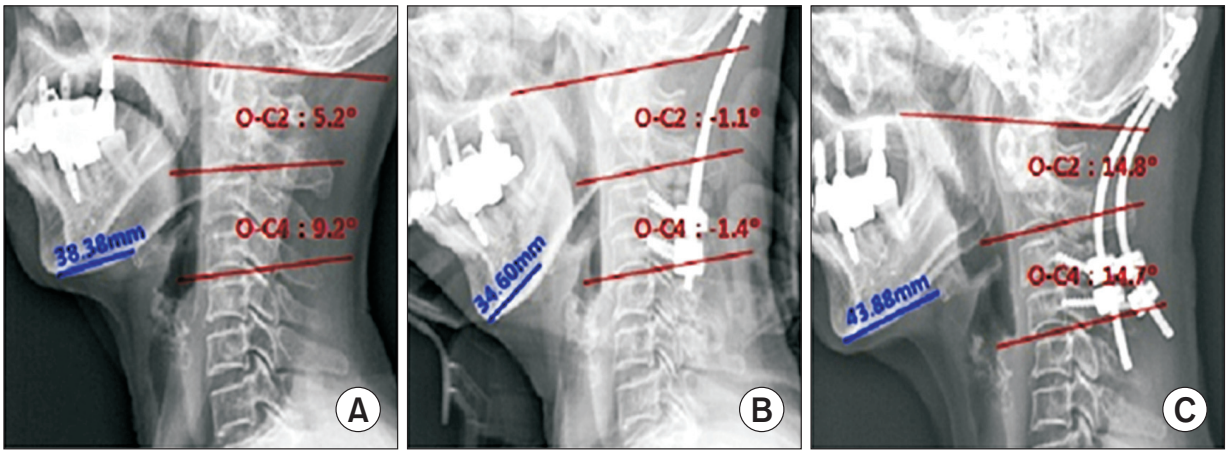

Fig. 1. Cervical spine lateral film measurements are progressively shown from left to right. (A) December 3, 2013 before the initial operation, (B) January 2, 2014 after the initial operation, and (C) March 27, 2014 after revision operation.

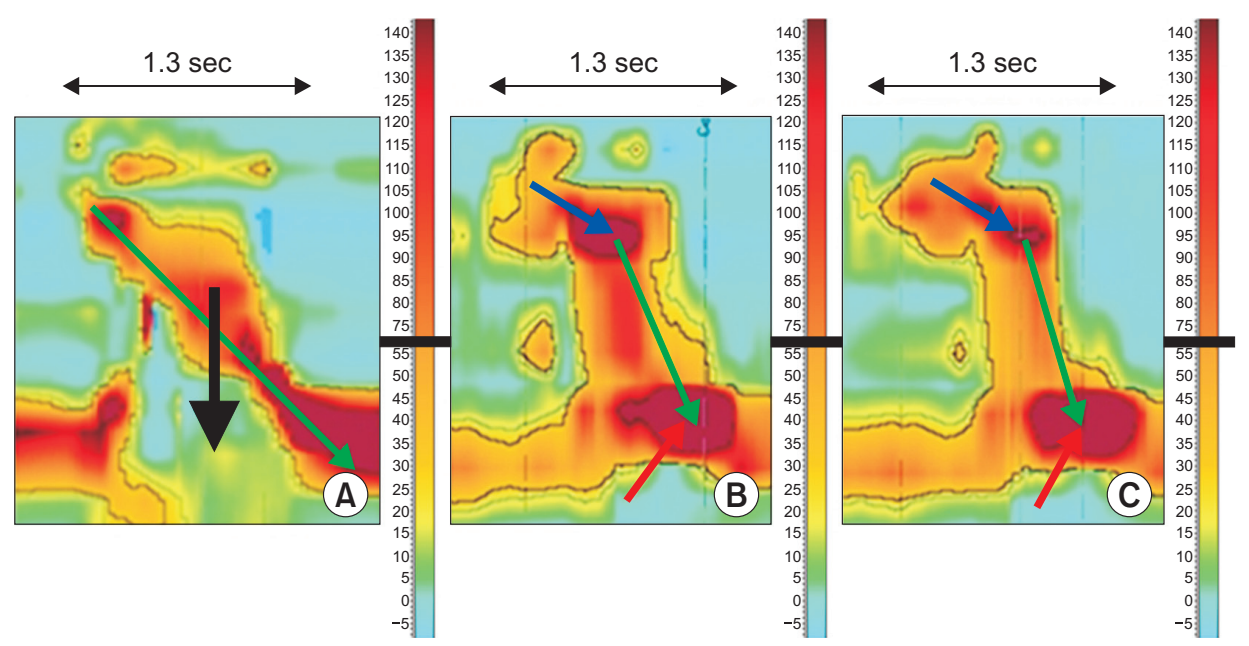

Fig. 2. Pharyngeal pressure generation patterns are shown: red reflects areas of high pressure and blue reflects areas of low pressure; the $\mathrm{X}$-axis represents time while the $\mathrm{Y}$-axis represents the pharyngeal location from the velopharynx to esophagus; sec indicates seconds while the numbers presented in the vertical column indicate the color representation of pressure (mmHg). The patterns were taken from a normal participant (A), the patient's dry swallow (B), and the patient's liquid swallow (C). Note the well-established high pressure in the rostral area and the low pressure in the caudal area in which the pressure gradient is produced during swallow in the normal sample (A, black arrow), which is not observable in the patient $(\mathrm{B}, \mathrm{C})$; the dark red area demonstrating high pressure at the upper esophageal sphincter (UES) zone implicates an impaired UES opening (B \& C, red arrow); the light red area at the velopharynx indicates good contraction forces (B \& C, blue arrow), but the peristaltic slope observable in the normal sample (A, green arrow) was diminished in the patient (B \& C, green arrow). 
pre-operative state and $-1.1^{\circ}$ in the post-operative state (Fig. 1A, B). The O-C4 angle changed from $9.2^{\circ}$ to $1.4^{\circ}$ (Fig. $1 \mathrm{~A}, \mathrm{~B})$. The change in $\mathrm{c}$-spine angle caused a shortening of the distance between the mental tubercle and the anterior border of the hyoid bone, which may indirectly reflect suprahyoid muscle distance. The distance was changed from $38.33 \mathrm{~mm}$ to $34.60 \mathrm{~mm}$, post-operatively.

In the VFSS study, laryngeal elevation was impaired and subglottic aspirations were observed for all viscosity levels during and after swallow. A large amount of residual material at the valleculae and pyriform sinuses was observed along with features of cricopharyngeal dysfunction.

Pharyngeal pressure generation parameters were acquired using a solid HRM that measures rapid changes in pressure along 1-cm spaced sensors (InSIGHT HRiM; Sandhill Scientific Instruments, Highlands Ranch, CO, USA) with the patient in the sitting position [5]. Spatiotemporal color mapping of pharyngeal pressure showed impaired upper esophageal sphincter (UES) opening along with diminished pressure gradient and peristaltic slope to be the most prominent problem (Fig. 2). The dark red area demonstrating high pressure at the UES zone implicates impaired UES opening (Fig. 2B, C). The light red area at the velopharynx and the tongue base (TB) indicates good contraction forces (Fig. 2B, C), but the peristaltic slope was diminished compared to the normal sample (Fig. 2A-C). Oropharyngeal contraction forces occurred simultaneously along all levels diminishing the pressure gradient, thereby interfering bolus propulsion (Fig. 2B, C).

Absolute values of the HRM parameters were also compared to the normative data ( 7 males and 3 females, age distribution $29.2 \pm 7.2$ years) acquired previously at our institution (Table 1). The maximal pressure and area of velopharynx were significantly lower while the maximal pressure of TB and UES minimal pressure were significantly higher. The pre-UES maximal pressure was significantly lower $(\mathrm{p}<0.05)$. The UES activity time and the nadir area were unobtainable due to the indistinguishable low pressure zone during swallow. However, the minimal

Table 1. Absolute values of pressure measurements and activation latencies

\begin{tabular}{|c|c|c|c|c|}
\hline \multirow{2}{*}{ Parameter } & \multicolumn{2}{|c|}{ Normal group } & \multicolumn{2}{|c|}{ Patient measurement } \\
\hline & Air & $5 \mathrm{~mL}$ & Air & $5 \mathrm{~mL}$ \\
\hline \multicolumn{5}{|l|}{ Velopharynx } \\
\hline Max pressure (mmHg) & $192.4 \pm 84.0$ & $232.5 \pm 107.7$ & $72.7^{\mathrm{a})}$ & $74.5^{\mathrm{a})}$ \\
\hline Area (mmHg.s) & $41.0 \pm 25.6$ & $49.6 \pm 32.8$ & $9.0^{\mathrm{a})}$ & $10.0^{\mathrm{a})}$ \\
\hline Rise time (s) & $0.2 \pm 0.2$ & $0.2 \pm 0.8$ & 0.1 & 0.2 \\
\hline Duration (s) & $0.3 \pm 0.2$ & $0.4 \pm 0.2$ & 0.2 & 0.3 \\
\hline \multicolumn{5}{|l|}{ Tongue base } \\
\hline Max pressure (mmHg) & $144.9 \pm 47.2$ & $142.1 \pm 30.3$ & $253.3^{a)}$ & $177.6^{\text {a) }}$ \\
\hline Area (mmHg.s) & $51.9 \pm 15.3$ & $53.0 \pm 16.8$ & $95.0^{\mathrm{a})}$ & 65.0 \\
\hline Rise time (s) & $0.3 \pm 0.1$ & $0.3 \pm 0.1$ & 0.3 & 0.4 \\
\hline Duration (s) & $0.6 \pm 0.1$ & $0.6 \pm 0.2$ & 0.6 & 0.6 \\
\hline \multicolumn{5}{|l|}{ Low pharynx } \\
\hline Max pressure (mmHg) & $450.8 \pm 133.3$ & $486.6 \pm 150.2$ & 524.5 & 481.5 \\
\hline \multicolumn{5}{|l|}{ UES } \\
\hline Pre-UES max pressure (mmHg) & $268.3 \pm 84.0$ & $240.6 \pm 119.6$ & $90.6^{\text {a) }}$ & $88.1^{\text {a) }}$ \\
\hline Post-UES max pressure (mmHg) & $377.3 \pm 129.4$ & $452.1 \pm 126.2$ & $121.3^{\mathrm{a})}$ & 338.5 \\
\hline UES minimal pressure $(\mathrm{mmHg})$ & $-11.65 \pm 9.10$ & $-6.21 \pm 5.43$ & $7.1^{\mathrm{a})}$ & $4.8^{\mathrm{a})}$ \\
\hline Activity time (s) & $0.7 \pm 0.2$ & $0.7 \pm 0.1$ & - & - \\
\hline Nadir duration (s) & $0.3 \pm 0.1$ & $0.4 \pm 0.1$ & - & - \\
\hline
\end{tabular}

Values are presented as mean \pm standard deviation.

Max, maximal; Min, minimal; Low, lower portion of pharynx $1 \mathrm{~cm}$ above the UES zone; UES, upper esophageal sphincter.

a) indicates observations more than 2 standard deviations different from the previously obtained normative values. 
pressure in the zone was obtainable and was found to be significantly lower than the high pressure zones, but still higher than the pressure of normal control patients (Table 1).

After evaluation, a nasogastric tube was inserted for patient safety. Operation for adjustment of the O-C angle was recommended. The patient received a revision operation on January 21,2014 . The O-C2 angle after revision was $14.8^{\circ}$ extended, and the $\mathrm{O}-\mathrm{C} 4$ angle was $14.7^{\circ}$ extended (Fig. 1C). The suprahyoid muscle distance increased from $34.60 \mathrm{~mm}$ to $43.88 \mathrm{~mm}$ (Fig. 1B, C). The patient was satisfied with the result and no longer complained of dysphagia. She is doing well with a regular diet and without any aspiration events.

\section{DISCUSSION}

Until now, there have been several studies regarding the mechanisms of dysphagia caused by O-C fusion [1-4]. These studies assessed the angle of $\mathrm{O}-\mathrm{C} 2$ and concluded that the change in cervical alignment after posterior fusion was the mechanism of dysphagia. One study evaluated a relationship between dysphagia prevalence and oropharyngeal airway space (PAS) revealing decreased PAS to be significantly associated to dysphagia [1]. However, these studies did not evaluate the actual pharyngeal pressure events, and therefore, their conclusions are limited.

This is the first report to verify the changes in pharyngeal pressure of dysphagia after O-C fusion using HRM. Our report shows that impaired UES opening, diminished pressure gradient for bolus propulsion, and impaired peristalsis from velopharynx to UES were the key problems in a patient with dysphagia caused by a change in cervical alignment after posterior fusion.

UES opening is currently explained by two mechanisms: 1) cessation of tonic activity at the cricopharyngeus and inferior pharyngeal constrictors and 2) active contraction of the suprahyoid muscles [6]. Mylohyoid, thyrohyoid, and geniohyoid muscles pull the cricoid cartilage superiorly and anteriorly, thus pulling the anterior wall of the UES along with it [6].

The patient received posterior fusion, which is known to have a much lower incidence of laryngeal neuropathy compared to anterior fusion [2]. The patient also did not have other persistent symptoms of laryngeal neuropathy. The absolute pressure values of HRM showed increased UES minimal pressure during swallow and unobtain- able nadir area, which is direct evidence of impaired UES opening (Table 1). However, the minimal pressure during swallow was obtainable and was significantly lower than normal UES tonic contraction pressure. Considering the fact that the patient was unlikely to have laryngeal neuropathy according to the clinical features, this finding may signify the existence of UES relaxation during swallow.

Therefore, the patient's dysfunction of the UES opening may be caused mainly by an insufficient pull of the suprahyoid muscles rather than impaired cessation of tonic UES activity. Currently, reports of change in cervical angle leading to change in mechanical properties of suprahyoid muscles are not available. However, muscle strength is known to decrease as muscle length is shortened from the optimal stretch distance [7]. Accordingly, it can be hypothesized that a change in cervical angle led to a change in the stretch distance of the suprahyoid muscles, which may have resulted in insufficient anterior pull during UES opening. Even though direct measurements of suprahyoid muscle distance are not available, the distance was indirectly measured through the c-spine lateral film (Fig. 1). The patient's suprahyoid muscle distance was shortened after posterior fusion, but recovered after the revision operation, which lengthened the shortened muscle distance from $34.60 \mathrm{~mm}$ to $43.88 \mathrm{~mm}$ (Fig. 1). The findings that the distance from the mental tubercle to the hyoid bone increased after the operation and that the symptoms subsided afterwards are in concordance with the hypothesis, though well designed studies are required in the future to verify this.

The second major problem of the patient was a diminished pressure gradient and peristalsis from velopharynx to UES. Intra-bolus pressure is highest in the bolus tail, while the intraluminal pressure distal to the bolus is of lower pressure, leading to generation of a propulsive pressure gradient [8]. A diminished propulsive pressure gradient under $20 \mathrm{mmHg}$ was reported to result in retrograde bolus escape, leading to ineffective propulsion of the contents [8]. The spatio-temporal mapping of HRM showed diffusely increased intraluminal pressure at the $\mathrm{TB}$ along with a steep slope of pressure generation, which is in concordance with the report above (Fig. 2). Decent TB contractile force alone, evidenced as increased absolute pressure of the TB region, was insufficient for decent peristalsis and bolus propulsion (Table 1). However, there are no reports available on the mechanism behind the re- 
lationship between cervical angle with loss of pharyngeal pressure gradient and peristalsis. The physiology behind the process remains to be verified in future studies.

As this report evaluated only one patient, further studies are required for generalization. Future studies should include more patients having developed dysphagia after $\mathrm{O}-\mathrm{C}$ fusion. Even though diminished pressure gradient, impaired peristalsis and impaired UES opening were the main observations acquired using the HRM, further studies on the underlying mechanism of such changes are required. Despite limitations, the HRM study was shown to have value by revealing a distinct pressure generation pattern of a patient with dysphagia caused by a change in cervical alignment, not recognizable with conventional measures.

\section{CONFLICT OF INTEREST}

No potential conflict of interest relevant to this article was reported.

\section{ACKNOWLEDGMENTS}

This research was supported by the Basic Science Research Program through the National Research Foundation of Korea (NRF) funded by the Ministry of Science, ICT and Future Planning (NRF-2013R1AlA1004622).

\section{REFERENCES}

1. Izeki M, Neo M, Takemoto M, Fujibayashi S, Ito H,
Nagai K, et al. The O-C2 angle established at occipitocervical fusion dictates the patient's destiny in terms of postoperative dyspnea and/or dysphagia. Eur Spine J 2014;23:328-36.

2. Tian $\mathrm{W}, \mathrm{Yu}$ J. The role of $\mathrm{C} 2-\mathrm{C} 7$ and $\mathrm{O}-\mathrm{C} 2$ angle in the development of dysphagia after cervical spine surgery. Dysphagia 2013;28:131-8.

3. Ota M, Neo M, Aoyama T, Ishizaki T, Fujibayashi S, Takemoto M, et al. Impact of the O-C2 angle on the oropharyngeal space in normal patients. Spine (Phila Pa 1976) 2011;36:E720-6.

4. Miyata M, Neo M, Fujibayashi S, Ito H, Takemoto M, Nakamura T. O-C2 angle as a predictor of dyspnea and/or dysphagia after occipitocervical fusion. Spine (Phila Pa 1976) 2009;34:184-8.

5. Fox MR, Bredenoord AJ. Oesophageal high-resolution manometry: moving from research into clinical practice. Gut 2008;57:405-23.

6. Cook IJ, Dodds WJ, Dantas RO, Massey B, Kern MK, Lang IM, et al. Opening mechanisms of the human upper esophageal sphincter. Am J Physiol 1989;257(5 Pt 1):G748-59.

7. Gordon AM, Huxley AF, Julian FJ. The variation in isometric tension with sarcomere length in vertebrate muscle fibres. J Physiol 1966;184:170-92.

8. Ren J, Massey BT, Dodds WJ, Kern MK, Brasseur JG, Shaker R, et al. Determinants of intrabolus pressure during esophageal peristaltic bolus transport. Am J Physiol 1993;264(3 Pt 1):G407-13. 